\title{
Enquêtes entomologiques préparatoires à une lutte à grande échelle contre les glossines, assistées par un système d'information géographique : cas de la Pattec au Burkina Faso
}

\author{
Lassané Percoma ${ }^{1}{ }^{*}$ Zowindé Koudougou ${ }^{1}$ \\ Oumarou Serdebeogo ${ }^{1}$ Issa Tamboura ${ }^{1}$ Mamadou Ouedraogo ${ }^{1}$ \\ Jérémy Bouyer ${ }^{2,3,4,5}$ Adrien M.G. Belem ${ }^{6}$ Issa Sidibé ${ }^{1,7}$
}

\begin{abstract}
Mots-clés
Glossina, lutte antiravageur, système d'information géographique, cartographie, entomologie, Burkina Faso
\end{abstract}

Accepted: 2 November 2015; Published: 9 May 2016
1. Pattec, BP 1087, 01 Bobo-Dioulasso, Burkina Faso.

2. Cirad, UMR Cmaee, Dakar-Hann, Sénégal.

3. Cirad, UMR Cmaee, 34398 Montpellier, France.

4. ISRA, Lnerv, Dakar-Hann, Sénégal.

5. Cirad, UMR Intertryp, 34398 Montpellier, France.

6. Université polytechnique de Bobo-Dioulasso, Burkina Faso.

7. Cirdes, 01 Bobo-Dioulasso, Burkina Faso.

* Auteur pour la correspondance

Tél. : + 226707392 06/769936 59; fax : + 22620970955 Email: percomalas@yahoo.fr

\section{Résumé}

Le système d'information géographique constitue une aide considérable lors de la définition d'un plan de lutte contre les glossines. Son application pratique pour la collecte de données entomologiques servant de base à la planification de la lutte et à son évaluation future a été faite dans l'ouest du Burkina Faso, de décembre 2007 à novembre 2008. Choisie pour englober les bassins versants des principaux fleuves ou rivières et les limites de distribution de Glossina palpalis gambiensis et G. tachinoides, la zone d'étude a été divisée en cellules de $10 \mathrm{~km} \times 10 \mathrm{~km}$. Dans chaque cellule, un maximum de 13 sites potentiels de piégeage ont été sélectionnés par I'utilisation d'imagerie satellite, puis repérés sur le terrain à l'aide du système mondial de positionnement (GPS). L'enquête entomologique a été réalisée avec des pièges biconiques Challier-Laveissière et les récoltes ont eu lieu 72 heures après leur installation. L'analyse des données a été conduite en utilisant des modèles aléatoires mixtes binomiaux et de distribution de Poisson. La cartographie, réalisée avec le logiciel Arc Gis 9.3, a permis de déterminer la limite nord $\left(12^{\circ} 45^{\prime} \mathrm{N}\right)$ des glossines dans la zone d'intervention. Les proportions de sites infestés étaient de 89,6 et $76,4 \%$ respectivement au niveau des sections ascendante et descendante de la rivière Mouhoun (la première coule vers le nord-est depuis la source, la seconde vers le sud-est puis le sud après avoir conflué avec le Sourou), et de 16,7 et 10,3\% respectivement au niveau de leurs affluents. Entre les isohyètes $700-800 \mathrm{~m}$ et 800 $900 \mathrm{~m}$ aucune différence significative n’a été observée dans les densités apparentes des glossines par piège, ni dans les sites infestés. Les deux espèces de glossines capturées étaient distribuées différemment selon les sections du Mouhoun : les captures étaient composées de 79,5\% de G. p. gambiensis sur la section ascendante et de $96,0 \%$ de G. tachinoides sur la section descendante. Les captures de vecteurs mécaniques ont été relativement faibles, leur distribution étant comparable à celle des glossines avec cependant quelques densités importantes sur les affluents.

- Comment citer cet article : Percoma L., Koudougou Z., Serdebeogo O., Tamboura I., Ouedraogo M. Bouyer J., Belem A.M.G., Sidibé I., 2015. Preparatory entomological surveys for full-scale tsetse-fly control , assisted with a geographic information system: PATTEC case in Burkina Faso [in French]. Rev. Elev. Med. Vet. Pays Trop., 68 (4): 157-165

\section{INTRODUCTION}

Les glossines ou mouches tsé-tsé sont les principaux vecteurs des trypanosomoses humaine et animales africaines, qui sont respectivement une maladie négligée et une des contraintes pathologiques majeures à la production animale en Afrique subsaharienne (Vreysen et al., 2011). Selon l'Organisation de l'unité africaine (OUA, 2000), les trypanosomoses constituent ainsi le plus grand obstacle à l'élevage et à la production agricole du continent. Considérées comme une grave menace pour la vie et les moyens de subsistance des communautés, elles coûtent annuellement plus de cinq milliards de dollars à l'économie africaine (Budd, 1999). 
Connues comme vecteurs des trypanosomoses depuis 1903, les tsétsé font l'objet d'une lutte acharnée dès les années 1920 (Laveissière et al., 2000). Les différentes méthodes de lutte employées ont permis de débarrasser des glossines de vastes zones au cours des années 1940, 1950 et 1960 dans plusieurs pays africains. Mais très rapidement, la plupart de ces acquis ont été amoindris du fait de la réinvasion (OUA, 2000)

A la lumière de ces expériences et consciente du fait que l'élimination de la mouche tsé-tsé et de la trypanosomose serait perçue comme un maillon important du redressement des économies africaines, l'Union Africaine a lancé en 2000, lors d'une réunion tenue à Lomé (Togo), une vaste campagne d'éradication (OUA, 2000). Solution idéale mais très difficile à atteindre selon Laveissière et al. (2000), l'eradication des glossines permettrait une amélioration des conditions de vie des ménages. L'approche stratégique retenue est le concept de area-wide integrated pest management. Cette approche consiste en une combinaison de plusieurs stratégies de lutte, les unes efficaces contre les populations de glossines de forte densité, les autres contre celles à faible densité, dans l'optique d'aboutir au contrôle ou à l'éradication totale dans une zone isolée, que ce soit naturellement ou par la pose de barrières (Vreysen et al., 2007 ; Vreysen et al., 2011).

La technique de l'insecte stérile est une grande composante de ce concept. Associée à plusieurs autres techniques, elle a été très souvent utilisée avec succès dans l'éradication, la suppression, ou le contrôle de diptères nuisibles dans divers pays. Le dernier exemple en date est l'éradication de Glossina austeni, Newstead, 1912, sur l'île de Zanzibar en 1997 (Vreysen et al., 2000 ; Vreysen et al., 2011).

Mais le choix d'une stratégie efficace de lutte visant l'éradication nécessite la détermination préalable de la distribution spatiale des populations cibles. Cette détermination est facilitée par l'intégration de l'exploitation des images satellites et d'un système d'information géographique (SIG). Dans le cadre de la campagne panafricaine d'éradication des tsé-tsé et des trypanosomoses (Pattec) au Burkina Faso, la zone d'intervention, correspondant à la zone infestée de glossines, a été délimitée à l'ouest du pays grâce à l'utilisation de ces techniques. L'objectif de l'étude a été de présenter la contribution du SIG à l'établissement de la carte de distribution des glossines dans la zone d'étude et de préciser les relations entre cette distribution et l'environnement afin de préparer la phase de lutte.

\section{MATERIEL ET METHODES}

\section{Choix et préparation de la zone d'étude}

La zone d'intervention de la Pattec Burkina se situe à l'ouest du pays sur une superficie de 96600 kilomètres carrés (figure 1). Elle a été délimitée en tenant compte des cartes prédictives de distribution de Glossina palpalis gambiensis (Gpg) et de Glossina tachinoides $(G t)$, et des bassins versants des principaux cours d'eau (Mouhoun principalement, et Bani venant du Mali) (Pattec, 2006). Le planning et la préparation de la campagne de lutte ont été faits selon la méthode de Leak et al. (2008) concernant la collecte des données entomologiques de base. La zone entière a été quadrillée par 966 cellules de $10 \mathrm{~km} \times 10 \mathrm{~km}$ (figure 1) et divisée en cinq blocs homogènes selon l'axe nord-sud.

Le bloc 1 a été retenu prioritairement pour effectuer les enquêtes préparatoires à la lutte du fait de l'existence d'une barrière naturelle de distribution de $G p g$ et $G t$ à sa limite nord. Mais par la suite la nécessité de trouver des sites facilement accessibles pour l'implantation d'autres barrières a conduit à l'intégration du bloc 2 dans la zone d'étude. Cette zone d'étude (soit les deux blocs réunis) a une superficie de 51300 kilomètres carrés. De plus, ce premier bloc couvre la boucle du Mouhoun qui se situe dans le triangle cotonnier ouest-africain, zone prioritaire pour la lutte contre les trypanosomoses animales africaines (Hendrickx et al., 2004 ; Bouyer, 2006).

\section{Traitement d'image satellitaire}

Des images satellitaires Landsat 2003 obtenues grâce au projet BKF5004 Feasibility Study on Applying the Sterile Insect Technique to Create a Tsetse free Zone, ainsi que d'autres images réalisées en 2000 ont été utilisées. Pour la sélection des sites de piégeage, une composition colorée rouge verte bleue (RGB) a été réalisée afin de mieux discriminer la végétation active. Une telle composition est la superposition de trois canaux colorés, chacun dans une couleur fondamentale, permettant d'identifier par des teintes contrastées les différents thèmes ou phénomènes recherchés (Girard et Girard, 1999 ; Guerrini, 2004). Un canal est une image (ou une information) obtenue pour chacune des régions du spectre électromagnétique capté par le satellite, qui permet de caractériser un objet au sol. La combinaison dite de fausses couleurs a été faite : la couleur rouge a été affectée à la végétation, le vert au sol nu et le bleu à l'eau. Dans le souci d'augmenter la résolution spatiale, la bande panchromatique a été utilisée avec les techniques resolution merge de Brovey Transform (Gillespie et al., 1987) et smoothing filter-based intensity modulation (SFIM) (Liu et Moore, 1998) pour obtenir une composition colorée de résolution 14,25 m au lieu de 28,5 m (Liu, 2000).

\section{Choix des sites de piégeage}

Des travaux antérieurs réalisés par la FAO, le Centre international de recherche-développement sur l'élevage en zone subhumide (Cirdes), l'Unité centrale de lutte contre la trypanosomiase / Agence internationale de l'énergie atomique (UCLT/AIEA) et la Direction du laboratoire nationale d'élevage (DLNE) ont permis de supprimer de la zone d'étude certaines parties de la province de la Kossi, où plusieurs enquêtes avaient révélé une absence de glossines. Pour le choix des sites de piégeage, la carte représentant les cellules de $10 \mathrm{~km}$ x $10 \mathrm{~km}$ a été superposée aux images satellitaires traitées. Les gîtes potentiels de présence des glossines étaient

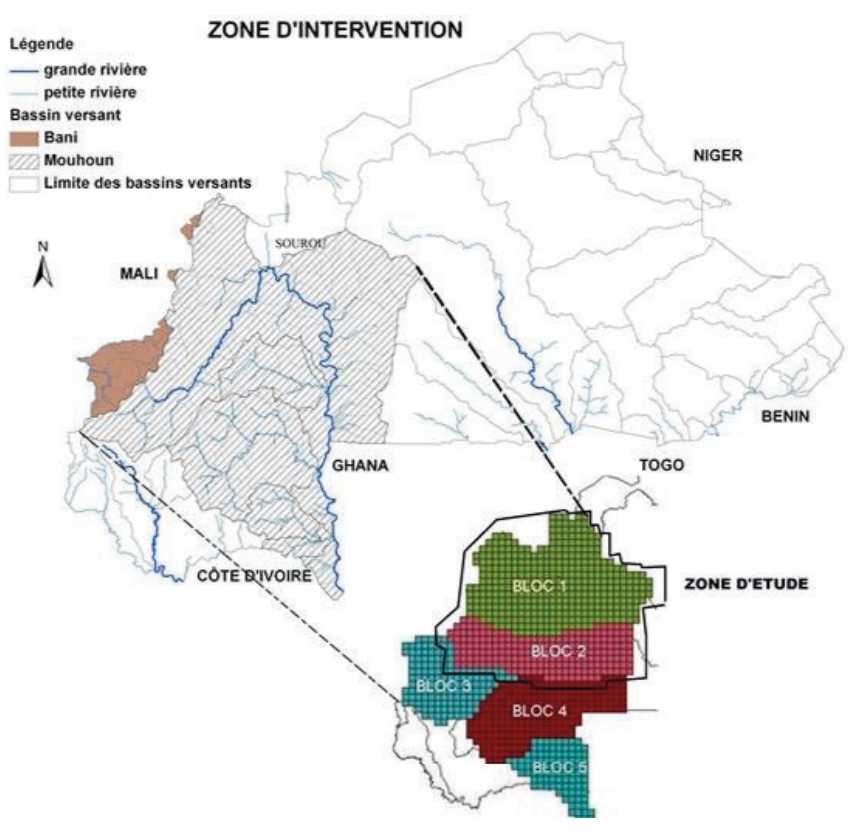

Figure 1 : zone d'étude située dans l'ouest du Burkina Faso. 
ceux ayant une forte densité de végétation et bénéficiant de la proximité de cours d'eau. Pour harmoniser l'enquête, un maximum de 13 sites a été retenu par cellule (figure 2). Les coordonnées des sites ainsi identifiées ont été transférées dans des GPS afin de guider les enquêtes entomologiques (Bouyer et al., 2010). Ces dernières ont été réalisées par quatre équipes qui ont évolué chacune sur une rive du Mouhoun du fait de la difficulté de traverser le cours principal et de prospecter simultanément de part et d'autre.

\section{Collecte de données entomologiques}

Sur la base des sites présélectionnés, des cartes thématiques ont été produites portant les codes des sites, les routes, les cours d'eau ainsi que les villages importants (villages riverains des sites). Avant la prospection, un important travail de sensibilisation sur l'impact socio-économique des glossines ainsi que sur les objectifs recherchés par l'initiative Pattec avait été fait auprès des chefs de villages, et des différentes communautés ethniques, religieuses, politiques et sociales (Rouamba et al., 2009). Sur le terrain, les sites ont été localisés grâce à l'utilisation de GPS. Les enquêtes ont été réalisées à l'aide de pièges biconiques (Challier et Laveissière, 1973) qui permettent d'obtenir un échantillon des populations de glossines représentatif d'une zone (Gouteux et al., 1981). Ce sont des pièges standards connus pour leur efficacité à capturer des glossines riveraines (Dao et al., 2008).

Dans chaque site, deux pièges distants de 100 mètres minimum ont été posés aux endroits considérés comme favorables : un maximum de 26 pièges ont donc été posés par cellule. Pour augmenter l'efficacité du piégeage, un sachet contenant $2 \mathrm{ml}$ d'une mixture d'attractif olfactif $(0,15 \mathrm{ml}$ de 3-n-propylphénol, 0,62 $\mathrm{ml}$ de 1-octén-3-ol et 1,23 ml de paracrésol) a été suspendu à un crochet à l'intérieur de chaque piège. L'adjonction de cet attractif olfactif multiplie par 4 à 5 les captures de Gt, et par 2 celles de $G p g$ (Rayaissé et al., 2010). Chaque piège a été géo-référencé et identifié par un code unique. Les données caractéristiques de localisation de chaque piège (région, province, département, terroir villageois, nom des membres de l'équipe, code du piège, longitude, latitude, altitude, date et heure de la pose, type de végétation,

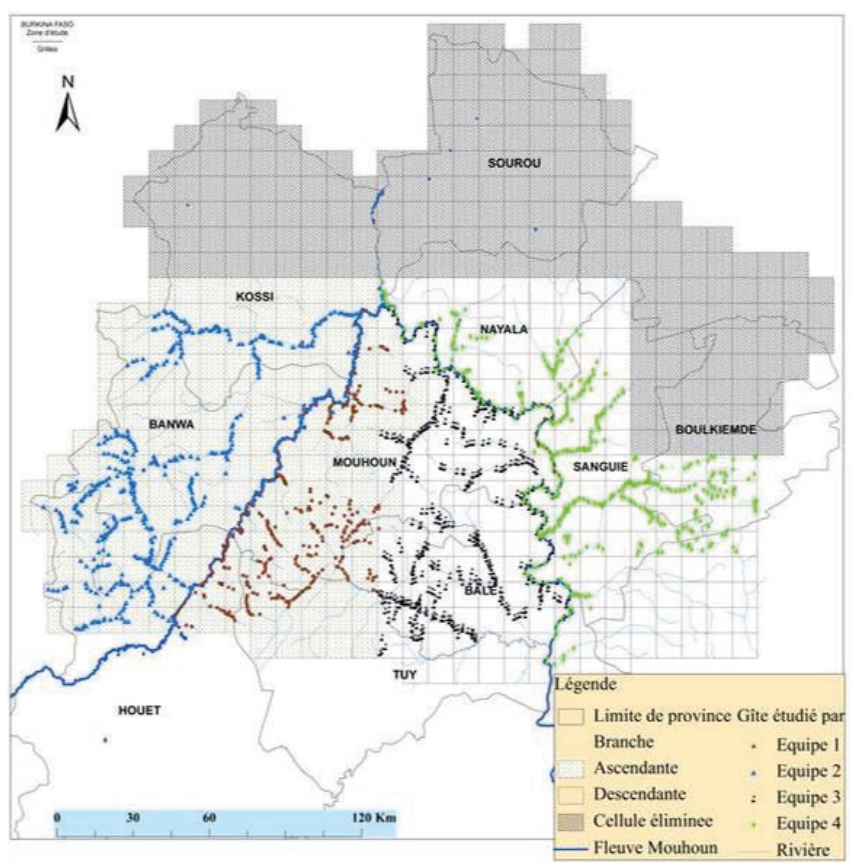

Figure 2 : distribution des sites de piégeage et des zones prospectées dans l'ouest du Burkina Faso par les quatre équipes. espèces d'arbres dominantes), ont été consignées sur des fiches d'enquête. La base du piquet de fixation a été enduite de graisse pour éviter la prédation des insectes capturés par des fourmis. Pour favoriser une bonne visibilité et éviter que les pièges ne soient brûlés par d'éventuels feux de brousse, l'herbe sèche autour d'eux était balayée.

Les pièges étaient récoltés 72 heures après leur installation et dans l'ordre de pose. Les glossines capturées étaient séparées par espèce et par sexe, et les vecteurs mécaniques l'étaient par famille ou sous-famille et dénombrés. La date et l'heure de récolte étaient relevées.

Les données présentées ci-dessous ont été récoltées en trois périodes. Débutée le 3 décembre 2007, la prospection dans le bloc 1 s'est achevée le 6 février 2008. Après une interruption nécessaire à la planification de la prospection dans le bloc 2 , cette dernière a débuté le 28 avril 2008, a été interrompue le 16 juin en raison de la saison pluvieuse qui rend l'accès aux sites impossible, et a repris le 10 novembre pour se terminer au cours du même mois.

\section{Analyse des données}

Deux grands critères environnementaux ont été pris en compte pour affiner la compréhension de l'écologie des glossines dans la zone d'étude : la localisation des pièges sur le cours d'eau principal (permanent) ou sur les affluents (en grande majorité temporaires), et la situation (amont/aval) par rapport au barrage du Sourou situé au sommet de la boucle du Mouhoun. La section ascendante du Mouhoun (partie coulant vers le nord-est, entre la source et la confluence avec le Sourou ; figure 1) possède en effet une forêt-galerie de type soudano-guinéen, alors que la section descendante (partie coulant vers le sud-est puis le sud après avoir conflué avec le Sourou) est de type soudanien (Bouyer et al., 2005). L'importance de ces critères pour expliquer la présence et l'abondance des glossines était connue mais devait être quantifiée pour optimiser la future campagne de lutte, en focalisant l'effort de lutte sur les zones les plus infestées.

L'analyse des pourcentages de sites infestés a été réalisée par des modèles aléatoires mixtes binomiaux et celle des densités apparentes par piège et par jour par des modèles aléatoires mixtes avec une distribution de Poisson. A chaque fois, le type de rivière près du piège (cours d'eau principal ou affluent) et sa position par rapport au Sourou (section ascendante ou descendante) ainsi que leur interaction de premier ordre ont été utilisés comme effets fixes. Les sites de piégeage ont été utilisés comme effets aléatoires. Les cartes ont été réalisées avec le logiciel Arc Gis 9.3.

\section{RESULTATS}

\section{Variation des taux d'infestation en glossines en fonction des sections du Mouhoun, des affluents, des isohyètes et des captures globales}

En 102 jours d'activité, 3189 pièges (trois pièges supplémentaires ont été posés dans trois sites différents) ont été posés dans 1593 sites, dont 833 sur la section ascendante du Mouhoun et 760 sur la section descendante. Des glossines ont été capturées dans 124 des 252 cellules prospectées (121 cellules ont été éliminées du fait qu'elles ne disposaient pas de cours d'eau et/ou de végétation favorable à l'existence de glossines ; figure 3). Au niveau de la section ascendante, $81,2 \%$ des pièges ont été posés sur des affluents ; la proportion a été de 79,2\% pour la section descendante. Le site positif situé le plus au nord a été observé à $12^{\circ} 45^{\prime} 51^{\prime \prime}$ de lat. N et $3^{\circ} 26^{\prime} 07^{\prime \prime}$ de long. O, sur le Sourou. De 
manière générale, 488 des 1593 sites prospectés (30,6 \%) étaient infestés de glossines, $32,9 \%$ des sites de la section ascendante et $28,2 \%$ de ceux de la section descendante.

Une plus faible proportion de sites était infestée sur les affluents que sur le cours principal du Mouhoun ( $\mathrm{p}<0,0001)$, et ce, sur les deux sections (figures 4 et 5). Seuls 19,9\% des sites des affluents de la section ascendante et 15,2\% des sites des affluents de la section descendante étaient infestés. Sur le cours principal, la section ascendante a présenté plus de sites infestés $(89,1 \%)$ que la section descendante $(76,4 \%)(\mathrm{p}=0,002)$.

Aucune différence significative n'a été observée entre les proportions de sites infestés pour les pièges posés entre les isohyètes 700 et $800 \mathrm{~mm}(89,7 \%$ de pièges positifs) et 800 et $900 \mathrm{~mm}(89,5 \%)$ au niveau du Mouhoun $(\mathrm{p}>0,96)$. Il n'y a pas eu non plus de

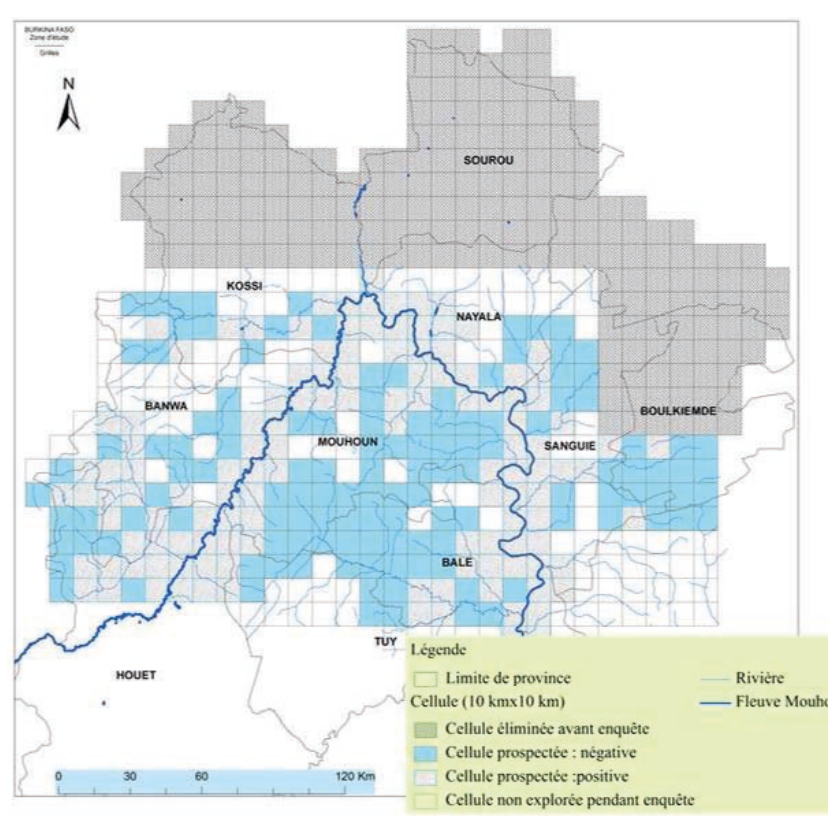

Figure 3 : cellules prospectées et non prospectées dans la zone d'étude située dans l'ouest du Burkina Faso.

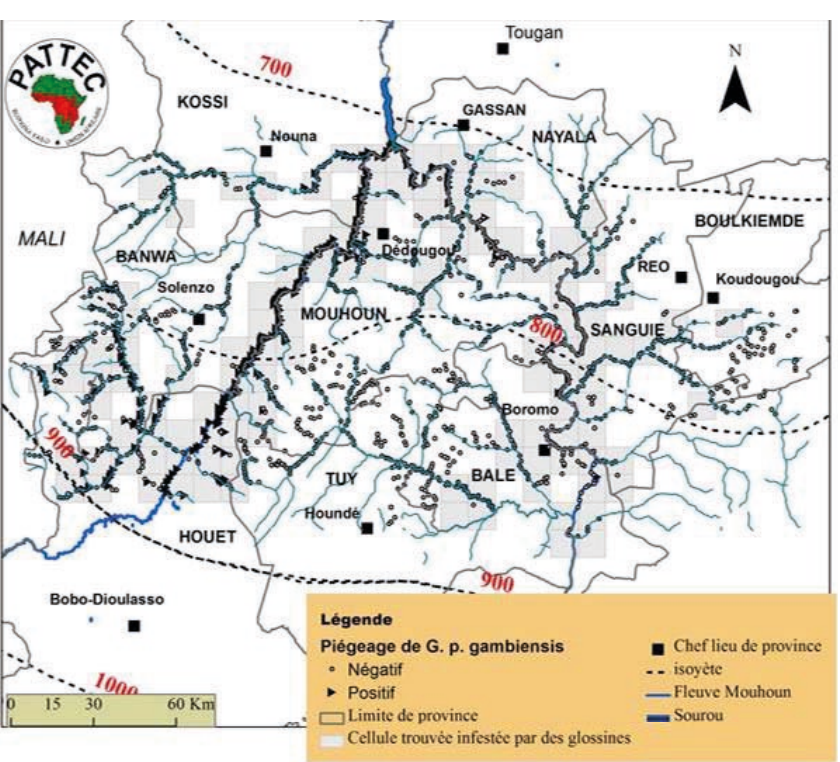

Figure 4 : répartition de Glossina palpalis gambiensis selon le cours d'eau et par niveau d'isohyète dans la zone de la rivière Mouhoun et de ses affluents au Burkina Faso.

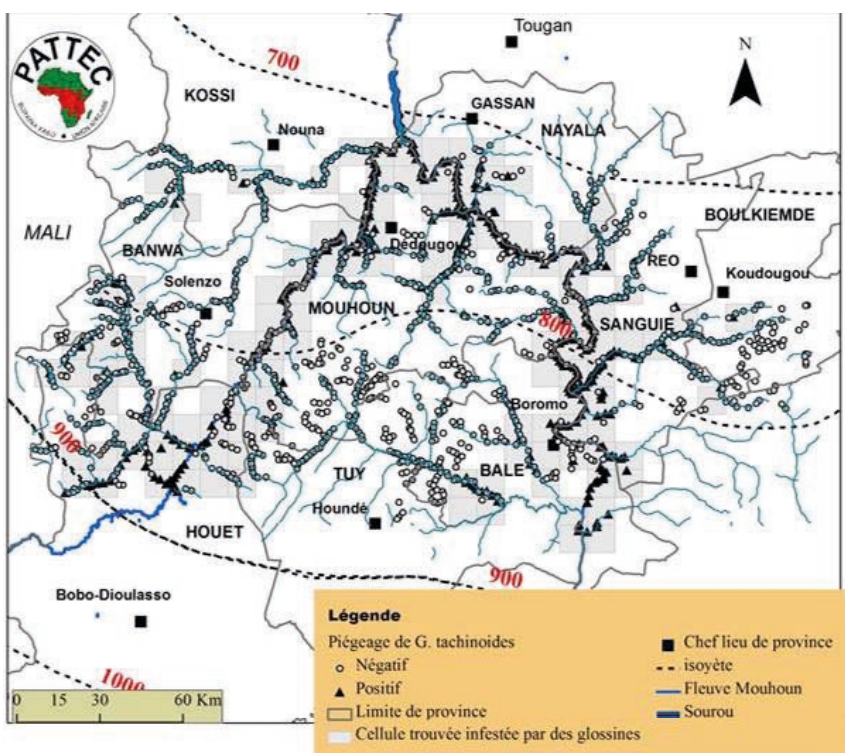

Figure 5 : répartition de Glossina tachinoides selon le cours d'eau et par niveau d'isohyète dans la zone de la rivière Mouhoun et de ses affluents au Burkina Faso.

différence significative entre les densités apparentes de glossines par piège par jour (DAP) selon les mêmes classes d'isohyètes, chaque section étant considérée séparément : les DAP moyennes ont été respectivement de 10,2 $\pm 1,0$ et 13,5 $\pm 1,6$ au niveau de la section ascendante, et de 2,2 $\pm 0,3$ et 3,7 $\pm 0,5$ au niveau de la section descendante. Le nombre de sites prospectés entre les isohyètes 600 et $700 \mathrm{~mm}$, et 900 et $1000 \mathrm{~mm}$ a été faible et ainsi n'a pas donné de résultats fiables.

\section{Importance relative des espèces de glossines le long des cours d'eau}

Deux espèces de glossines, Gpg et Gt, ont été identifiées avec une grande variabilité selon les sections du Mouhoun. Gpg a été capturée dans 86,2 $\pm 4,7 \%$ des sites de la section ascendante et $15,7 \pm 12,5 \%$ de ceux de la section descendante, et $G t$ respectivement dans $47,0 \pm 18,1 \%$ et $75,9 \pm 0,6 \%$ des sites. Au total, 16972 glossines ont été capturées dont $9348 \mathrm{Gpg}$ et $7624 \mathrm{Gt}$ (tableau I). Les sex-ratios ont été respectivement de 1,1 et 0,8 pour Gpg et Gt.

Sur la section ascendante du Mouhoun, 98,83 \% de Gpg et $33,68 \%$ de $G t$ ont été capturées ; ces proportions ont été respectivement de $1,17 \%$ et $66,12 \%$ sur la section descendante. La section ascendante a été plus infestée que la section descendante ( $\mathrm{p}<0,0001)$ : 10279 glossines y ont été capturées dans 312 pièges contre 2600 glossines dans 319 pièges sur la section descendante (tableau I).

En ce qui concerne les DAP, 92 Gpg et 43,3 Gt ont été observées au maximum sur la section ascendante du Mouhoun entre les isohyètes 700 et $800 \mathrm{~mm}$, et 5,3 Gpg et 108,7 Gt sur la section descendante, entre les isohyètes 800 et $900 \mathrm{~mm}$. Sur la section ascendante, plus on s'éloignait du Sourou, plus le nombre de Gpg augmentait et celui de $G t$ baissait. Le contraire a été observé au niveau de la section descendante.

\section{Vecteurs mécaniques}

Seules la sous-famille des Stomoxyinae et la famille des Tabanidae ont été identifiées. Au total, 8513 vecteurs mécaniques ont été capturés par 1417 des 3189 pièges posés (44,4\%). De manière générale, les densités moyennes par piège et par jour ont été faibles 
aussi bien sur le cours principal que sur les affluents (tableau II). Une DAP maximale de 72 tabanidés a toutefois été notée sur un affluent de la section ascendante. Les vecteurs mécaniques ont surtout été présents en novembre-janvier, après la saison pluvieuse.
Sur les deux sections, une dominance des tabanidés par rapport aux stomoxes $(\mathrm{p}<0,05)$ a été relevée dans les captures. Les DAP moyennes ont été de $1,1 \pm 3,5$ tabanidés et $0,1 \pm 0,6$ stomoxe sur la section ascendante, et de $0,5 \pm 1,8$ tabanidés contre $0,02 \pm 0,2$

\section{Tableau I}

Taux d'infestation et densités apparentes des glossines au niveau de la rivière Mouhoun et de ses affluents au Burkina Faso

\begin{tabular}{|c|c|c|c|c|c|c|}
\hline & & \multicolumn{2}{|c|}{ Pièges } & \multicolumn{3}{|c|}{ Glossines } \\
\hline & & Total & $\%$ positif & Total & $\begin{array}{c}\text { DAP } \\
\text { maximale }\end{array}$ & $\begin{array}{c}\text { DAP } \\
\text { moyenne }\end{array}$ \\
\hline \multicolumn{7}{|c|}{ Glossina palpalis gambiensis } \\
\hline \multirow[t]{2}{*}{ Section ascendante } & Mouhoun & 312 & 77 & 8151 & 92,0 & $8,7 \pm 0,7$ \\
\hline & Affluents & 1344 & 10 & 1088 & 33,3 & $0,3 \pm 0,1$ \\
\hline \multirow[t]{2}{*}{ Section descendante } & Mouhoun & 319 & 11,3 & 103 & 5,3 & $0,1 \pm 0,0$ \\
\hline & Affluents & 1214 & 0,2 & 6 & 1,7 & $0,002 \pm 0,0$ \\
\hline Total/moyenne & & 3189 & & 9348 & & $2,3 \pm 1,2$ \\
\hline \multicolumn{7}{|l|}{ Glossina tachinoides } \\
\hline \multirow[t]{2}{*}{ Section ascendante } & Mouhoun & 312 & 43,0 & 2128 & 43,3 & $2,3 \pm 0,3$ \\
\hline & Affluents & 1344 & 6,4 & 455 & 19,3 & $0,1 \pm 0,0$ \\
\hline \multirow[t]{2}{*}{ Section descendante } & Mouhoun & 319 & 63,0 & 2497 & 26,3 & $2,6 \pm 0,3$ \\
\hline & Affluents & 1214 & 11,5 & 2544 & 108,7 & $0,7 \pm 0,2$ \\
\hline Total/moyenne & & 3189 & & 7624 & & $1,4 \pm 0,2$ \\
\hline \multicolumn{7}{|c|}{ Glossina palpalis gambiensis et G. tachinoides } \\
\hline \multirow[t]{2}{*}{ Section ascendante } & Mouhoun & 312 & 81,4 & 10279 & 120,0 & $11,0 \pm 0,8$ \\
\hline & Affluents & 1344 & 14,7 & 1543 & 36,0 & $0,4 \pm 0,1$ \\
\hline \multirow[t]{2}{*}{ Section descendante } & Mouhoun & 319 & 64,6 & 2600 & 26,3 & $2,7 \pm 0,3$ \\
\hline & Affluents & 1214 & 11,5 & 2550 & 108,7 & $0,7 \pm 0,2$ \\
\hline Total/moyenne & & 3189 & & 16972 & & $3,7 \pm 0,3$ \\
\hline
\end{tabular}

\section{Tableau II}

Taux d'infestation et densités apparentes des vecteurs mécaniques au niveau de la rivière Mouhoun et de ses affluents au Burkina Faso

\begin{tabular}{|c|c|c|c|c|c|c|}
\hline & & \multicolumn{2}{|c|}{ Pièges } & \multicolumn{3}{|c|}{ Vecteurs mécaniques } \\
\hline & & Total & $\%$ positif & Total & $\begin{array}{c}\text { DAP } \\
\text { maximale }\end{array}$ & $\begin{array}{c}\text { DAP } \\
\text { moyenne }\end{array}$ \\
\hline \multicolumn{7}{|l|}{ Stomoxes } \\
\hline \multirow{2}{*}{ Section ascendante } & Mouhoun & 312 & 17,3 & 141 & 3 & $0,2 \pm 0,0$ \\
\hline & Affluents & 1344 & 7,1 & 317 & 14 & $0,1 \pm 0,0$ \\
\hline \multirow{2}{*}{ Section descendante } & Mouhoun & 319 & 5,3 & 40 & 4 & $0,04 \pm 0,01$ \\
\hline & Affluents & 1214 & 1,9 & 67 & 6 & $0,02 \pm 0,0$ \\
\hline Total/moyenne & & 3189 & $7,9 \pm 6,6$ & 565 & & $0,1 \pm 0,1$ \\
\hline \multicolumn{7}{|l|}{ Tabanidés } \\
\hline \multirow[t]{2}{*}{ Section ascendante } & Mouhoun & 312 & 53,5 & 1459 & 29 & $1,6 \pm 0,2$ \\
\hline & Affluents & 1344 & 47,3 & 4095 & 72 & $1,02 \pm 0,1$ \\
\hline \multirow[t]{2}{*}{ Section descendante } & Mouhoun & 319 & 49,2 & 919 & 34 & $1,0 \pm 0,2$ \\
\hline & Affluents & 1214 & 34,2 & 1475 & 24 & $0,4 \pm 0,04$ \\
\hline Total/moyenne & & 3189 & $46,0 \pm 8,3$ & 7948 & & $1,0 \pm 0,5$ \\
\hline
\end{tabular}


stomoxe sur la section descendante. Comme chez les glossines, les vecteurs mécaniques ont été davantage présents sur le Mouhoun que sur ses affluents ( $p=0,035$ pour les Stomoxyinae et $p=0,013$ pour les Tabanidae).

\section{- DISCUSSION}

L'utilisation du SIG a permis un gain économique substantiel. Son intérêt en entomologie médicale et vétérinaire avait déjà été signalé par Laveissière et al. (2000). En effet, aidant à circonscrire la zone d'intervention et à rallier les sites présélectionnés pour la prospection, il a permis de réduire de moitié la durée impartie à l'activité de collecte de données entomologiques sur le terrain dans le plan d'action. L'utilisation des images satellites a aussi permis une bonne harmonisation et une bonne représentativité des sites prospectés dans la zone d'intervention. Bon outil de planification, le SIG a permis à la fin des enquêtes une bonne cartographie de la distribution des deux espèces de glossines, indispensable à la planification de la lutte.

Une durée de capture des glossines de 72 heures a été suffisante pour juger de l'absence totale ou temporaire des glossines au niveau du site de capture, mais il aurait été intéressant que l'activité soit reproduite à différentes saisons. Les enquêtes ont toutefois été réalisées à une période où les glossines étaient confinées dans leurs gîtes favorables le long des cours d'eau (saison sèche).

Au niveau des bassins des sections ascendante et descendante du Mouhoun, respectivement 81,3 et 78,8 \% des sites prospectés étaient sur ses affluents. La présence de glossines sur le Mouhoun avait déjà été mentionnée par les précédentes études de Guerrini (2004) et Bouyer (2006). Le travail majeur de cette enquête devait donc être effectué sur les affluents pour obtenir une connaissance complète de la situation sur la zone d'intervention.

Malgré le nombre considérable de sites prospectés au niveau des affluents, une proportion relativement faible s'est révélée infestée de glossines alors que plus de $75 \%$ des sites étaient infestés au niveau du cours d'eau principal. Ce faible nombre serait dû aux facteurs environnementaux (température, humidité, présence/absence d'hôtes nourriciers, végétation) qui rendraient ces sites peu favorables à la survie des glossines dans la plupart des affluents, notamment du fait qu'ils sont en majorité temporaires. Le tarissement de ces cours d'eau pendant les six mois de saison sèche, associé aux fortes températures, provoque certainement une grande mortalité des pupes enfouies dans le sol. De plus, les hôtes des espèces $G p g$ et $G t$ riveraines étant inféodés à l'eau, leur rareté pendant la saison sèche, du fait du manque d'eau, serait fatale aux glossines (Challier, 1973). Pour Laveissière et al. (2000), la conjonction d'un ou de plusieurs facteurs biotiques et abiotiques, comme un climat inadéquat, une pluviosité trop faible, une végétation inappropriée, l'absence d'hôte nourricier, a des conséquences sur la distribution spatiale des tsé-tsé car les glossines du groupe palpalis (dont font partie Gpg et Gt) sont principalement confinées aux régions très humides : les marais à palétuviers, les forêts ombrophiles, les rives des lacs et les forêts galeries longeant les rivières (Pollock, 1982).

D'un point de vue épidémiologique, cette distribution des glossines engendre un risque trypanosomien plus élevé au niveau du Mouhoun (Bouyer, 2006) qui est utilisé pour la pêche, comme zone de pâture et d'abreuvement des animaux, ou pour des cultures maraichères. Ceci a été corroboré par Sow et al. (2013) qui ont trouvé que la prévalence des trypanosomoses animales était plus élevée chez les animaux des villages riverains du Mouhoun lors d'une étude intégrant 53 localités de la boucle du Mouhoun.
Seules deux espèces de glossines, Gpg et $G t$, ont été identifiées dans la zone, comme rapporté par Guerrini (2004) et Bouyer (2006). Glossina morsitans submorsitans semble y avoir disparu du fait de l'accroissement démographique observé après les indépendances et de la forte anthropisation de la zone. Depuis lors, les principaux vecteurs de la trypanosomose animale sont donc ces deux espèces, plus résistantes aux changements d'origine anthropique (Van den Bossche et al., 2010). Au Burkina Faso, la boucle du Mouhoun est connue comme étant un foyer historique de la trypanosomiase humaine africaine (THA) et des trypanosomoses animales africaines. De nombreux villages localisés à proximité des cours d'eau ont autrefois disparu du fait de la THA associée parfois à l'onchocercose (Gouzien, 1908).

La limite nord de distribution des deux espèces de glossines capturées était située sur le Sourou, à $12^{\circ} 45^{\prime} \mathrm{N}$. Challier et Laveissière (1977) avaient établi la limite de distribution des glossines, pour le nord-ouest du Burkina, au niveau du $13^{\mathrm{e}}$ parallèle pour $G p g$ et à $13^{\circ} 17^{\prime} \mathrm{N}$ pour Gt. Les lieux de captures les plus septentrionaux avaient été trouvés dans la région de Tougan. A l'ouest du sommet de la boucle de la Volta noire, actuel Mouhoun, la limite était à $12^{\circ} 40^{\prime} \mathrm{N}$. Par conséquent, on note une descente de la limite nord dans la zone d'étude d'une trentaine de kilomètres. Cette descente s'expliquerait par plusieurs facteurs. D'une part, l'aménagement de la vallée du Sourou en 1983 a favorisé une anthropisation importante de la zone où les actions médicales de l'équipe de Muraz a permis en 1939 la stérilisation des réservoirs humains de trypanosomes (Rouamba et al., 2009). D'autre part, il y a les changements climatiques actuels, faisant suite à la sécheresse des années 1970 (Laveissière, 1976), et la dégradation continue du couvert végétal qui ont pour corollaire la disparition des glossines.

Selon Courtin et al. (2009), la limite nord des glossines se situe actuellement en Afrique de l'Ouest, au niveau de Dakar et de Ouagadougou, soit une descente d'environ 200 kilomètres par rapport aux résultats des piégeages de Bouët-Roubaud au siècle dernier. Mais cette descente est moindre dans la boucle du Mouhoun. Cela s'expliquerait par la présence de la rivière et de son affluent, le Sourou, qui entretiennent des conditions de température, d'hygrométrie et d'ambiance favorables aux hôtes nécessaires à la survie des glossines. De plus, contrairement à $G . m$. submorsitans inféodée à la faune sauvage, Gpg et $G t$ sont des espèces opportunistes qui s'adaptent facilement aux fortes densités de populations humaines (Courtin et al., 2009).

Ce sont sans doute ces mêmes raisons qui expliquent qu'il n'y ait pas de différence significative entre, d'une part, les proportions de sites infestés et, d'autre part, les densités de glossines en fonction des isohyètes au niveau du cours principal. Ainsi, la DAP la plus importante de $G t(108,7)$ a été observée au niveau de la section descendante sur un affluent, entre les isohyètes 800 et $900 \mathrm{~mm}$. Sur la section ascendante, c'est sur le cours principal et entre les isohyètes 700 et $800 \mathrm{~mm}$ que les DAP maximales (92 Gpg et 43,3 $G t)$ ont été notées. Ces résultats confirment l'hypothèse de Bouyer et al. (2005) selon laquelle aucune relation n'existe entre les densités apparentes de glossines sur le Mouhoun et la pluviométrie annuelle.

On a observé une distribution spatiale différente des deux espèces. Sur le Mouhoun, elles étaient présentes sur la section ascendante dans un rapport de 3,8 Gpg pour $1 \mathrm{Gt}$ alors que ce rapport était en faveur de $G t$ sur la section descendante, avec 24,2 contre 1. Etant donné que l'abondance ou la rareté de la faune sauvage, déterminées par ses migrations saisonnières, n'ont aucune importance sur ces tsé-tsé comparé à la variation des conditions physiques de leurs habitats (Challier, 1973), cette distribution selon les sections s'expliquait par la spécificité de chacune d'elles pour un certain type 
de végétation ripicole. Nos travaux confirment ainsi à nouveau la théorie de Morel (1978) selon laquelle Gpg préfère les galeries guinéennes fermées, $G t$ les galeries ouvertes soudaniennes, et que l'on retrouve les deux dans les galeries soudano-guinéennes (Bouyer et al., 2005). Gt préfère ainsi une végétation plus ouverte, principalement arbustive (Gruvel, 1975). Dans l'étude de l'influence de l'anthropisation sur la végétation locale et l'abondance des tsé-tsé au sud du Burkina Faso, Rayaissé et al. (2009) ont également noté que la galerie de type soudanien sur une portion de la Comoé était particulièrement favorable à $G t$ qui s'y trouvait en très fortes densités, et beaucoup moins à $G p g$ qui préférait les galeries de type guinéen.

Les glossines ont été capturées selon un rapport de 3,9 pour 1 sur respectivement les sections ascendante et descendante du cours principal. Les moindres captures faites sur la section descendante pourraient être dues à l'inondation des pupes, déposées dans les berges des rivières. En effet, comparé à la section ascendante, le lit de cette section du Mouhoun est peu profond. Durant la saison pluvieuse, on assiste ainsi à un important débordement pendant plus de quatre mois. En conséquence, les pupes déjà déposées sont noyées.

Comme chez les glossines, il y avait plus de sites infestés par les vecteurs mécaniques de la trypanosomose animale sur le Mouhoun que sur ses affluents. Par ailleurs, plus de tabanidés que de stomoxes ont été capturés. Koné et al. (2011) ont également noté la prédominance des tabanidés, représentant 93,2\% des captures dans cette zone. De manière générale, les moyennes des DAP ont été faibles. Les captures auraient été plus importantes si les pièges Nzi, pièges de référence pour les tabanidés en Afrique, avaient été utilisés dans notre étude (Mihok, 2002 ; Acapovi et al., 2001 ; Desquesnes et al., 2005). Les plus fortes prévalences observées sur le cours principal étaient dues au fait qu'il offrait les conditions environnementales nécessaires à la vie de ces insectes.

La connaissance de la distribution spatiale des vecteurs cycliques et mécaniques est un atout considérable pour la lutte à grande échelle. Pour que cette lutte soit efficace et durable elle doit intégrer toute la zone infestée. Toutefois, plus d'attention devrait être accordée au Mouhoun qui accueille l'essentiel des glossines et qui est comparable à une autoroute pour les réinfestations, c'est-àdire qu'il constitue un excellent corridor génétique (Bouyer et al., 2007). Un maximum de moyens devrait, d'autre part, être mis en place sur la section ascendante qui est la plus infestée en glossines. Malheureusement, ses berges sont parfois difficiles d'accès, ce qui peut constituer une entrave sérieuse à l'éradication.

\section{CONCLUSION}

L'utilisation d'un système d'information géographique comme moyen de planification a permis un gain substantiel de temps et une bonne harmonisation des sites prospectés. Ce système a, en outre, permis une cartographie de la distribution des deux espèces de glossines riveraines capturées, ce qui est un bon moyen d'orientation de la lutte. Les deux espèces se localisent majoritairement au niveau du Mouhoun qui offre des conditions optimales à leur survie. Dans le cadre d'une lutte, l'essentiel des actions devrait donc être mené au niveau de cette rivière. Malgré les changements climatiques (sécheresse accentuée) et la forte anthropisation, la descente du front nord de la limite de distribution des glossines n'est pas très importante dans la boucle du Mouhoun. Elle est d'environ 30 kilomètres par rapport à sa position en 1976.

Il y a une variation certaine de la distribution des glossines au niveau du Mouhoun. Au niveau de la section ascendante, Gpg et
Gt cohabitent mais avec une prédominance de la première sur la deuxième. Sur la section descendante, Gpg est presque absente. D'une manière générale, les glossines colonisent le cours du Mouhoun sur toute sa longueur. Cependant l'existence d'une barrière naturelle au nord de $12^{\circ} 45^{\prime} \mathrm{N}$ offre des possibilités pour l'application d'une lutte à grande échelle contre les glossines à condition que des barrières soient mises en place au sud des zones assainies.

Ainsi, un système dit de tapis roulant pourrait être appliqué avec la construction de barrières au fur et à mesure de l'avancée de la lutte à partir du premier site infesté localisé sur le Sourou, en remontant la section ascendante, d'une part, et en descendant l'autre section, d'autre part. Mais le voisinage avec le Mali, le Ghana, le Bénin, le Togo et la Côte d'Ivoire nécessite une coordination régionale sans laquelle les résultats obtenus ne pourront être durables.

\section{Remerciements}

Nous remercions la FAO, le Cirdes, l'UCLT, l'AIEA et la DLNE pour leurs appuis multiformes. Ils ont en outre permis l'acquisition de données de qualité sur la limite nord de distribution de Glossina palpalis gambiensis et de G. tachinoides ainsi que celle des images satellitaires pour la planification des activités. Nous exprimons notre gratitude à l'AIEA pour l'appui en matériel informatique et logistique et de cartographie. Nos remerciements vont également aux communautés rurales qui n'ont ménagé aucun effort pour nous accompagner dans toutes les localités, même les plus interdites (bois sacrés).

\section{REFERENCES}

Acapovi G.L., Yao Y., N’Goran E., Dia M.L., Desquesnes M., 2001. Relative abundance of tabanids in the savanna regions of Côte d'lvoire [in French]. Rev. Elev. Med. Vet. Pays Trop., 54 (2): 109-114

Bouyer J., 2006. Ecologie des glossines du Mouhoun au Burkina Faso : intérêt pour l'épidémiologie et le contrôle des trypanosomoses africaines. Thèse Doct., Université Montpellier 2, Montpellier, France, 204 p.

Bouyer J., Guerrini L., César J., de La Rocque S., Cuisance D., 2005. A phyto-sociological analysis of the distribution of riverine tsetse flies in Burkina Faso. Med. Vet. Entomol., 19 (4): 372-378, doi: 10.1111/j.1365-2915.2005.00584.x

Bouyer J., Ravel S., Dujardin J.-P., de Meeûs T., Vial L., Thévenon S., Guerrini L., Sidibé I., Solano P., 2007. Population structuring of Glossina palpalis gambiensis (Diptera: Glossinidae) According to landscape fragmentation in the Mouhoun River, Burkina Faso. J. Med. Entomol., 44 (5): 788-795, doi: 10.1093/jmedent/44.5.788

Bouyer J., Seck M.T., Sall B., Ndiaye E.Y., Guerrini L., Vreysen M.J.B. 2010. Stratified entomological sampling in preparation of an areawide integrated pest management program: The example of Glossina palpalis gambiensis (Diptera: Glossinidae) in the Niayes of Senegal. J. Med. Entomol., 47 (4): 543-552, doi: 10.1093/jmedent/47.4.543

Budd L.T., 1999. DFID-funded tsetse and trypanosomiasis research since 1980. Vol. 2: Economic analysis. Natural Resources International, Aylesford, UK, $123 \mathrm{p}$.

Challier A., 1973. Ecologie de Glossina palpalis gambiensis Vanderplank, 1949 (Diptera - Muscidae) en savane d'Afrique occidentale. Orstom, Paris, France, 290 p. (Mém. Orstom n 64)

Challier A., Laveissière C., 1973. Un nouveau piège pour la capture des glossines (Glossina : Diptera, Muscidae) : description et essais sur le terrain. Cah. Orstom, Sér. Entomol. Méd. Parasitol., 11 (4) : 251-262

Challier A., Laveissière C., 1977. La répartition des glossines en HauteVolta : carte à $1 / 2000000$. Notice explicative $n^{\circ}$ 69. Orstom, Paris, France, $34 \mathrm{p}$. 
Courtin F., Sidibé I., Rouamba J., Jamonneau V., Gouro A., Solano P., 2009. Population growth and global warming: impacts on tsetse and trypanosomoses in West Africa [in French]. Parasite, 16 (1): 3-10, doi: 10.1051/parasite/2009161003

Dao B., Hendrickx G., Sidibé I., Belem A.M.G., de La Rocque S., 2008. Impact of drought and degradation of protected areas on the distribution of bovine trypanosomoses and their vectors in the Ot catchment basin of Northern Togo [in French]. Rev. Elev. Med. Vet. Pays Trop., 61 (3-4): 153-160

Desquesnes M., Dia M.L., Acapovi G., Yoni W., 2005. Les vecteurs mécaniques des trypanosomoses animales. Généralités, morphologie, biologie impacts et contrôle. Identification des espèces les plus abondantes en Afrique de I'Ouest. Cirdes, Bobo-Dioulasso, Burkina Faso, $70 \mathrm{p}$.

Gillespie A.R., Kahle A.B., Walker R.E., 1987. Color enhancement of highly correlated images. II. Channel ratio and "chromaticity" transformation techniques. Remote Sens. Environ., 22 (3): 343-365, doi: 10.1016/0034-4257(87)90088-5

Girard M.-C., Girard C.M., 1999. Traitement des données de télédétection. Dunod, Paris, France, 529 p.

Guerrini L., 2004. Discrimination des paysages du Mouhoun et distribution des glossines riveraines. Mémoire DEA, Université d'Avignon, France, 39 p.

Gouteux J.-P., Challier A., Laveissière C., 1981. Modifications et essais du piège à glossines (Diptera, Glossinidae) Challier-Laveissière. Cah Orstom, Sér. Entomol. Méd. Parasitol., 19 (2): 87-99

Gouzien P., 1908. La maladie du sommeil dans le Haut-Sénégal et Niger. Document technique $\mathrm{n}^{\circ}$ 490. OCCGE, Bobo-Dioulasso, Burkina Faso, 25 p.

Gruvel J., 1975. General data on the ecology of Glossina tachinoides Westwood 1850 in the Kalamaloué reserve, lower Chari River valley [In French]. Rev. Elev. Med. Vet. Pays Trop., 28 (1): 27-40

Hendrickx G., de La Rocque S., Mattioli R.C., 2004. Long-term tsetse and trypanosomiasis management options in West Africa. FAO, Rome, Italy, $57 \mathrm{p}$.

Koné N., N'Goran E.K., Sidibé I., Kombassere A.W., Bouyer J., 2011. Spatio-temporal distribution of tsetse and other biting flies in the Mouhoun River basin, Burkina Faso. Med. Vet. Entomol., 25 (2): 156168, doi: 10.1111/j.1365-2915.2010.00938.x

Laveissière C., 1976. Répartition des glossines en Haute-Volta : effets de la grande sécheresse de 1972-1973. Cah. Orstom, Sér. Entomol. Méd. Parasitol., 14 (4) : 293-299

Laveissière C., Grébaut P., Herder S., Penchenier L., 2000. Les glossines vectrices de la trypanosomiase humaine africaine. IRD, Oceac, Yanoudé, Cameroun, 246 p.

Leak S.G.A., Ejigu D., Vreysen M.J.B., 2008. Collection of entomological baseline data for tsetse area-wide integrated pest management programmes. FAO, Rome, Italy, 207 p.

Liu J.G., 2000. Smoothing filter-based intensity modulation: a spectral preserve image fusion technique for improving spatial details. Int. J. Remote Sens., 21 (18): 3461-3472, doi: 10.1080/014311600750037499
Liu J.G., Moore J.McM., 1998. Pixel block intensity modulation: adding spatial detail to TM band 6 thermal imagery. Int. J. Remote Sens., 19 (13): 2477-2491, doi: 10.1080/014311698214578

Mihok S., 2002. The development of a multipurpose trap (the Nzi) for tsetse and other biting flies. Bull. Entomol. Res., 92 (5): 385-403, doi: 10.1079/BER2002186

Morel P.C., 1978. Les arbres et arbustes des savanes ouest-africaines (document pour l'étude de l'écologie des glossines), vol. 1. GTZ, Eschborn, Allemagne, $75 \mathrm{p}$.

Organisation de l'Unité africaine, 2000. Plan d'action continental pour l'éradication de la mouche tsé-tsé et de la trypanosomiase. OUA, Addis-Abbeba, Ethiopie, 30 p.

PATTEC, 2006. Plan d'action détaillé pour la collecte des données entomologiques de base dans le bassin du Mouhoun. PATTEC, BoboDioulasso, Burkina Faso, 67 p.

Pollock J.N., 1982. Manuel de lutte contre la mouche tsé-tsé. Vol. 1 : Biologie, systématique et répartition des tsé-tsé. FAO, Rome, Italie, $310 \mathrm{p}$.

Rayaissé J.-B., Courtin F., Akoundjin M., César J., Solano P., 2009. Influence of anthropisation on local vegetation and tsetse abundance in southern Burkina Faso [in French]. Parasite, 16 (1): 21-28, doi: 10.1051/parasite/2009161021

Rayaissé J.-B., Tirados I., Kaba D., Dewhirst S.Y., Logan J.G., Diarrassouba A., Salou E., Omolo M.O., Solano P., Lehane M.J., Pickett J.A., Vale G.A., Torr S.J., Esterhuizen J., 2010. Prospects for the development of odour baits to control the tsetse flies Glossina tachinoides and G. palpalis s.I. PLoS Negl. Trop. Dis., 4 (3): e632, doi: 10.1371/journal.pntd.0000632

Rouamba J., Jamonneau V., Sidibé I., Solano P., Courtin F., 2009. Impact of the dynamics of human settlement on tsetse and trypanosomosis distribution in the Mouhoun River basin (Burkina Faso) [in French]. Parasite, 16 (1): 11-19, doi: 10.1051/parasite/2009161011

Sow A., Ganaba R., Percoma L., Sidibé I., Bengaly Z., Adam Y., Koné P., Sawadogo G.J., Van Den Abbeele J., Marcotty T., Delespaux V., 2013. Baseline survey of animal trypanosomosis in the region of the Boucle du Mouhoun, Burkina Faso. Res. Vet. Sci., 94 (3): 573-578, doi: 10.1016/j.rvsc.2012.12.011

Van den Bossche P., de La Rocque S., Hendrickx G., Bouyer J., 2010. A changing environment and the epidemiology of tsetse-transmitted livestock trypanosomiasis. Trends Parasitol., 26 (5): 236-243, doi: 10.1016/j.pt.2010.02.010

Vreysen M.J.B., Robinson A.S., Hendrichs J., 2007. Area-wide control of insect pests: from research to field implementation. Springer, Dordrecht, Netherlands, 789 p.

Vreysen M.J.B., Saleh K.M., Ali M.Y., Abdulla A.M., Zhu Z.-R., Juma K.G., Dyck A., Msangi A.R., Mkonyi P.A., Feldmann H.U., 2000. Glossina austeni (Diptera: Glossinidae) eradicated on the island of Unguja, Zanzibar, using the sterile insect technique. J. Econ. Entomol., 93 (1): 123-135, doi: 10.1603/0022-0493-93.1.123

Vreysen M.J.B., Saleh K.M., Lancelot R., Bouyer J., 2011. Factory tsetse flies must behave like wild flies: A prerequisite for the sterile insect technique. PLoS Negl. Trop. Dis., 5 (2): e907, doi: 10.1371/journal. pntd.0000907 


\section{Summary}

Percoma L., Koudougou Z., Serdebeogo O., Tamboura I., Ouedraogo M., Bouyer J., Belem A.M.G., Sidibé I. Preparatory entomological surveys for full-scale tsetse-fly control, assisted with a geographic information system: PATTEC case in Burkina Faso

A geographic information system is a helpful tool for the definition of a control plan against tsetse. Its practical application in entomological data collection for the planning and future evaluation of tsetse control was carried out in Western Burkina Faso from December 2007 to November 2008. The sampling area was selected according to the main rivers basins and the limits of Glossina palpalis gambiensis and G. tachinoides distributions. The study area was gridded in 100-kilometer-square cells. In each cell a maximum of 13 potential trapping sites were selected, then spotted in the field by using a global positioning system. The entomological survey was carried out with biconical Challier-Laveissière traps collected 72 hours after their installation. The data analysis was performed with binomial mixed random and Poisson distribution models. Mapping was carried out with Arc GIS 9.3 to help to determine the Northern limit $\left(12^{\circ} 45^{\prime} \mathrm{N}\right)$ of tsetse distribution in the target area. The proportions of infested sites were 89.6 and $76.4 \%$ on the ascending and descending sections of Mouhoun River, respectively (the first flows northeastward from the spring, and the second southeastward, then southward from the confluence with Sourou River), and 16.7 and $10.3 \%$ on their tributaries, respectively. Between isohyets 700-800 and 800-900 m no significant differences were observed in tsetse apparent density per trap or in the infested sites. Both trapped species were however distributed unevenly depending on the sections of Mouhoun River. On the ascending branch G. p. gambiensis represented $79.5 \%$ of tsetse catches, and on the descending branch G. tachinoides represented $96.0 \%$ of them. Catches of mechanical vectors were relatively few. Their distributions were similar to that of tsetse but with higher densities on some tributaries.

Keywords: Glossina, pest control, geographic information system, cartography, entomology, Burkina Faso

\section{Resumen}

Percoma L., Koudougou Z., Serdebeogo O., Tamboura I., Ouedraogo M., Bouyer J., Belem A.M.G., Sidibé I. Encuestas entomológicas preparatorias para el control a larga escala de la mosca tse-tsé, con ayuda de un sistema de información geográfica: caso PATTEC en Burkina Faso

Un sistema de información geográfica es un instrumento útil para la definición de un plan de control contra la tse-tsé. Se llevó a cabo su aplicación práctica para la recolecta de datos entomológicos para el planeamiento y la evaluación futura del control de tse-tsé en Burkina Faso del Oeste, entre diciembre 2007 y noviembre 2008. La zona de muestreo se seleccionó de acuerdo a los principales lechos fluviales y a los límites de distribución de Glossina palpalis gambiensis y G. tachinoides. El área de estudio se cuadrilló en celdas de 100 kilómetros cuadrados. En cada celda se seleccionaron un máximo de 13 potenciales sitios de trampas, encontrados en el campo mediante el uso de un sistema de posicionamiento global. La encuesta entomológica se llevó a cabo con trampas bicónicas de Challier-Laveissière, recolectadas 72 horas después de la instalación. El análisis de datos se realizó mediante el modelo al azar mixto binomial y de distribución de Poisson. El mapeo se llevó a cabo con Arc GIS 9.3 con el fin de ayudar a determinar el límite norte $\left(12^{\circ} 45^{\prime} \mathrm{N}\right)$ de la distribución de la tse-tsé en el área de enfoque. Las proporciones de los sitios infestados fue de 89,6 y $76,4 \%$ en las ramas ascendente y descendente del río Mounhoun respectivamente (las primeras corrientes orientadas noreste desde la naciente y la segunda sudeste, luego hacia el sur del confluente con el río Sourou), y 16,7 y $10,3 \%$ de sus afluentes respectivamente. Entre isohietas 700-800 y 800-900 m, no se observaron diferencias significativas en la densidad aparente de la mosca tse-tsé, ni por trampa ni en los sitios infestados. Sin embargo, las dos especies atrapadas fueron distribuidas de forma desigual en función de las ramas del río Mouhoun. En la rama ascendente, G. p. gambiensis representó el 79,5\% de las capturas de la mosca tse-tsé , y en la rama descendente $G$. tachinoides representó el 96,0 \% de ellos. Las capturas de vectores mecánicos fueron relativamente pocas. Sus distribuciones fueron similares a la de la mosca tse-tsé, pero con densidades más altas en algunos afluentes.

Palabras clave: Glossina, control de plagas, sistema de información geográfica, cartografía, entomología, Burkina Faso 
